\title{
The multiscale simulation of apical myocardial infarction and shape variation of the left ventricle of the heart
}

\author{
Fyodor Syomin ${ }^{1,2, *}$, Albina Khabibullina ${ }^{3}$, Anna Osepyan $^{3}$, and Andrey Tsaturyan ${ }^{1}$ \\ ${ }^{1}$ Institute of Mechanics, Lomonosov Moscow State University, 1 Mitchurinski pr-t, Moscow, 119192, \\ Russian Federation \\ ${ }^{2}$ Peoples' Friendship University of Russia (RUDN University), 6 Miklukho-Maklaya St, Moscow, \\ 117198, Russian Federation \\ ${ }^{3}$ Faculty of Mechanics and Mathematics, Lomonosov Moscow State University, 1 Leninskie Gory, \\ Moscow, 119991, Russian Federation
}

\begin{abstract}
A multiscale model of the cardiovascular system (CVS) in which the left ventricle (LV) of the heart was approximated by an axisymmetrical thickwall body made of transversely isotropic incompressible material was used to simulate the performance of the heart with apical myocardial infarction (MI). The material model reproduced mechanical properties and calcium regulation of active tension in cardiac muscle. The changes in the LV strain and the reduction of the LV stroke volume and arterial blood pressure obtained in the MI simulations were similar to those observed in patients with the apical MI. In contrast to the decrease in heart performance in the MI simulations, the simulation of changes in the LV shape from "normal" to a spherical or conical one revealed only slight changes in haemodynamics provided that the LV preload and the mass of the LV wall were kept constant.
\end{abstract}

\section{Introduction}

Mathematical modelling is a useful tool for understanding how the heart works in health and disease [1]. Multiscale models could be used in clinics for more detailed diagnostics and the choice of adequate treatment of heart diseases. In particular, myocardial infarction (MI) is a heart disease of interest for computer simulation [2]. Earlier we had developed a model of the cardiovascular system with a focus on the left ventricle (LV) of the heart [3]. The LV was represented by a multiscale model describing the macroscopic pumping function of the ventricle considering the dependence of stress-strain relationships at the tissue level on the microscopic processes at the cell level [4]. The advantage of the model compared to those with a simplistic description of myocardium mechanics is a detailed description of the interactions of contractile and regulatory proteins in cardiac cells [5], that is important for accurate simulation of the heart mechanics.

Our multiscale model of the LV within CVS described by a lumped parameter model reproduces the time-courses of haemodynamic variables (pressures in the LV, aorta, veins, volume of the LV) for an averaged "healthy" patient, and the effects of some arrhythmias and heart valve diseases [3]. Here we report the result of simulation of the effects of apical

\footnotetext{
*e-mail: f.syomin@imec.msu.ru
} 
MI on the LV performance and geometry during a heartbeat. Some heart diseases, including ischemic and non-ischemic LV dilatation and MI, are often accompanied by changes in the LV shape. Surgical ventricular restoration (SRV) is often used to restore the elliptical shape of LV in patients with MI [6] resulting in an increase in the ventricular performance reportedly. On the contrary, the decrease in heart performance was reported for an early postoperative period after SVR of apical MI resulting in more spherical shapes of LVs [7, 8]. The question whether the LV shape changes per se affect the LV performance and should be eliminated surgically is under discussion $[6,7,9]$. To address this question, we simulated the effects of changes in the LV shape at the same mass and mechanical properties of the LV wall on the LV function. The LV shape was characterized by the sphericity and conicity indices used in clinical practice [10].

\section{Methods}

\subsection{Mathematical model of the LV within CVS}

The key element of our CVS model is a multiscale model of the LV. The ventricle was approximated by a body of rotation close to a semi-ellipsoid [11]. Every point of the ventricular wall in the rectangular coordinate plane was associated with curvilinear coordinates $\gamma$ (the point position across the wall equal zero at subendocardium and one at subepicardium) and $\psi$ (the point angular position equal zero at the base of the LV and $\pi / 2$ at its apex). The corresponding equations were

$$
\begin{aligned}
& x=\left(r_{\text {in }}+\gamma \cdot\left(r_{\text {out }}-r_{\text {in }}\right)\right) \cdot(\varepsilon \cos \psi+(1-\varepsilon)(1-\sin \psi)), \\
& y=\left(h_{\text {in }}+\gamma \cdot h_{\text {apex }}\right) \cdot(1-\sin \psi)+(1-\gamma) \cdot h_{\text {apex }} .
\end{aligned}
$$

Here, parameters $r_{\text {in }}, r_{\text {out }}, h_{\text {in }}, h_{\text {apex }}$, and $\varepsilon$ set a preliminary end-systolic geometry, in particular, inner and outer radii at the base, the axial size of the ventricular cavity, the thickness of the apex, and a variation in the curvature of the wall (the shape is described by a cone at $\varepsilon$ equals zero).

The myocardium of the LV wall was assumed to be incompressible transversely isotropic material in that non-isotropic stress of axial forces applied along the fibres of cardiac muscle was described by our kinetic model [5]. The kinetic model was set by a system of ordinary differential equations (ODEs) for variables determining several states of contractile and regulatory proteins. In all numerical experiments presented in the paper, the myocardium fibres were oriented in a spiral manner with helical angle changing from $80^{\circ}$ at the inner LV wall to $-55^{\circ}$ at the outer wall.

The other three chambers of the heart, as well as systemic and pulmonary circulations, were described by a new lumped parameter model, which was also set by ODEs for pressures $P$ and volumes $V$ of several segments of the vascular bed, considered as elastic reservoirs, and for the flows $Q$ between them. Inertial and hydraulic flow resistances $(R$ and $L)$ and compliances $C$ of each reservoir were assumed to be constant parameters. The general forms of the equations were

$$
C \frac{d P}{d t}=Q_{i n 1}-Q_{o u t 1}, \frac{d V}{d t}=Q_{i n 2}-Q_{o u t 2}, L \frac{d Q}{d t}+R \cdot Q=P_{1}-P_{2} .
$$

Atria and the right ventricle were treated as viscoelastic bodies pressure-volume relationships for which included an activation function depended on time and the chamber volume. The equations for the pressures caused by an active stress in the walls of the chambers had the 
following form

$$
\begin{aligned}
& \tau \frac{d P}{d t}+P=F_{A c t} \cdot\left(\mu \frac{d V}{d t}+E_{A c t}\right), \\
& F_{A c t}=e_{A c t}(t) \cdot\left(k_{V}+\left(1-k_{V}\right) \cdot\left(\frac{V-V_{\min }}{V_{\max }-V_{\min }}\right)\right) .
\end{aligned}
$$

The first expression in 3 is an analogue of Force-Velocity relationship for the muscle with non-dimensional force $F_{A c t}$ and maximal isovolumic steady-state pressure $E_{A c t}$. The second expression states how $F_{A c t}$ depended on activation function $e_{A c t}$ and, in order to reproduce Frank's law of the heart, on the chamber volume. The lumped parameter model was described in our recent paper [3].

The equilibrium equations for the LV were related to the lumped parameter model by the volume of the LV cavity $V_{L V}$ and LV pressure $P_{L V}$, which was included in boundary conditions for the inner wall of the ventricle. Some reasonable initial conditions for the lumped parameter model and the kinetic model of muscle contraction were chosen. The LV contractions were initiated by the periodic simultaneous influx of calcium ions to the cells of the ventricular wall and had been performed until the steady periodical solution had been obtained. The details of the model and its numerical implementation are given in our previous works $[3,4]$.

\subsection{Algorithm of calculation of the shape indices}

The shape indices were calculated by an algorithm suggested in [10]. Both sphericity and conicity indices $S_{i}$ were set by the expression $S_{i}=1-A / A_{\text {max }}$, where $A$ is an area between the curves of shape description signals $\rho_{L V}$ and $\rho_{\text {Ref }}$ which were plotted on a normalized angular coordinate (zero at the ventricular apex, one at the base). In our case of axisymmetric geometry, the functions $\rho_{L V}$ and $\rho_{R e f}$ had simplified forms. The first function is the distance from the ventricular axis to the point at the endocardial surface normalized by its maximal value and multiplied by the halved aspect ratio of the LV equatorial cross section; the second one is the signal of the reference shape that is the hemisphere with the radius of 0.5 and the centre at $(0.5,0)$, for the sphericity index, or it is the straight line $y=a \cdot x$ for the conicity index ( $a=r / h$ is a halved aspect ratio of the ventricle). $A_{\max }$ is an area of the whole coordinate plane equal to 0.5 by default. The end-diastolic coordinates of the inner nodes of our simulated LV were used for $\rho_{L V}$ calculations. Due to the axial symmetry we could use normalized longitudinal coordinates of the nodes as the parameter of $\rho_{L V}$ instead of angular coordinates.

\section{Results and discussion}

\subsection{Apical myocardial infarction}

Our model allows us to simulate local defects in properties of the LV myocardium. The most straightforward dysfunction that can be simulated is a regional axisymmetric myocardium infarction (MI). The simulated apical MI was set by a local decrease in the additional factor $F_{\text {inf }}$ (multiplier of the active tension) in the apical region as follows

$$
F_{\text {inf }}=\left\{\begin{array}{l}
1, \psi<\psi_{1} \\
k_{\text {inf }}+\left(1-k_{\text {inf }}\right) \cdot\left(\frac{\psi_{2}-\psi}{\psi_{2}-\psi_{1}}\right)^{2}, \psi_{1} \leq \psi \leq \psi_{2} \\
k_{\text {inf }}, \psi>\psi_{2}
\end{array}\right.
$$

The passive mechanical properties of the LV myocardium were remained unchanged. Simulation of the apical infarction was performed with the values of the parameters $k_{\text {inf }}, \psi_{1}, \psi_{2}$ 
being equal zero, $\pi / 4$ and $3 \pi / 10$, respectively. Relative volume of the infarcted tissue was near $14 \%$ of the total volume of the ventricular wall.

The end-systolic and end-diastolic shapes of the normal and MI LVs with colour maps for sarcomere length are shown in figure 1 . The results for the MI LV demonstrate significantly

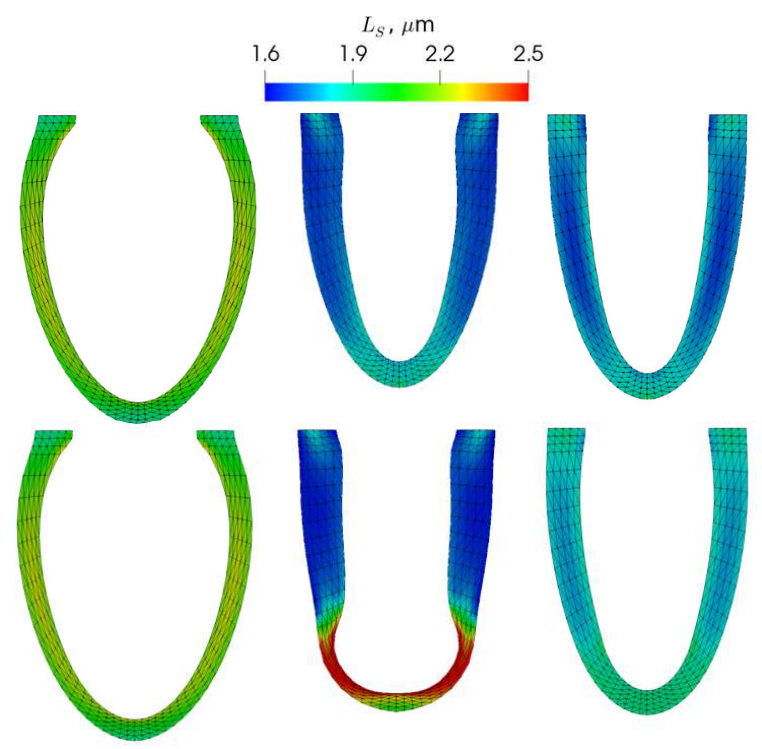

Figure 1. The shape and sarcomere length for the models of "normal" LV (top) and the LV with apical MI (bottom). End-diastolic (left), end-systolic (middle) LVs and the shape at the beginning of diastole (right) are shown

non-uniform fibre strains in the apical region at the end of systole including, in particular, abnormally large strains of endocardial fibres at the apex. One of the clinical parameters for estimating ventricular dysfunction is so called global longitudinal strain (GLS). In medical practice GLS is measured by tracking the deformations of several segments of the ventricular wall in an ultrasound image [12]. It was found out that GLS is usually decreased in patients with heart failure, and the GLS measurements are more reliable than measurements of ejection fraction [13] for the prediction of the heart failure. A correlation of GLS with MI size was reported [14], and the mean MI size of $15 \%$ corresponded to the mean GLS of $-13 \%$ while normal GLS was assumed to be equal $-20 \%$. Again MI size had better correlation with GLS than with ejection fraction. The results of our simulation of the MI LV also demonstrate a notable decrease in magnitude of GLS (-9\%) compared to the "healthy" LV $(-17 \%)$.

The resulting time-courses of the main haemodynamic variables, presented in figure 2 , show impaired ventricular performance: decreases in stroke volume (from 81 to $72 \mathrm{~mL}$ ), ejection fraction (from 64\% to 56\%) and maximal pressures in the LV (from 130 to $117 \mathrm{~mm} \mathrm{Hg}$ ) and aorta (from 122 to $109 \mathrm{~mm} \mathrm{Hg}$ ). However, the decrease in heart performance was not very significant considering quite wide region of MI. That might be caused by limitations of our model. It seems that the presence of the right ventricle and its connection to the LV in the apical region could be crucial for stress and strain distribution in the region. Moreover, our current model does not consider possible remodelling of the LV after MI commonly observed in medical practice. In particular, it was estimated both experimentally and numerically that the MI region becomes stiffer and the distribution of the cardiac fibres orientation changes 


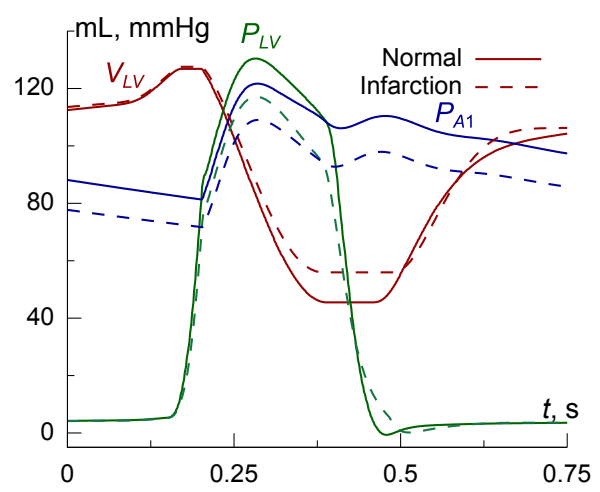

Figure 2. Simulated haemodynamics of the LV with apical MI. The time-courses of the LV volume, pressure, and aortic pressure for the "normal" LV (solid lines) and for the MI LV (dash lines) are presented

Table 1. Shape indices of the simulated LVs

\begin{tabular}{cccc}
\hline & "Normal" & Spherical & Conical \\
\hline Sphericity index & 0.73 & $\mathbf{0 . 8 3}$ & 0.58 \\
Conicity index & 0.76 & 0.58 & $\mathbf{0 . 8 4}$ \\
\hline
\end{tabular}

significantly under the remodelling [15]. These changes in the myocardial stiffness and fibre orientation were not taken into account in our model.

\subsection{Effects of LV shape}

We have performed simulations to examine numerically the dependence of the LV performance on its shape. Geometrical parameters $r_{\text {in }}, r_{\text {out }}, h_{\text {in }}$, and $h_{\text {apex }}$ were set up in such way that the cavity volume and the wall thickness of the LV preliminary end-systolic geometry would be the same as they were for the "normal" LV, while the relation of the radius of the ventricular cavity to its length would change drastically. Then we scaled the whole geometry to obtain the "normal" values of the volume of the ventricular wall. We also slightly changed initial preload (initial pressure values in pulmonary and systemic veins) in order to obtain 'healthy' end-diastolic LV pressures observer in simulations of the "normal" LV. The endsystolic and end-diastolic geometries of the spherical and conical LVs with a colour legend for sarcomere lengths are shown in figure 3. These results demonstrate less uniform distribution of fibre strain across the ventricular wall for the LVs with high conicity or sphericity indices than for the "normal" one. The sarcomeres shortened more significantly during systole of the spherical LV, while the subendocardial fibres underwent larger deformations during diastole. For conical LV one can notice less uniform end-systolic distribution of sarcomere lengths and very small strains in the apex. That means, considering length-dependent activation of the muscle, that muscle fibers in the middle-part equatorial region of the conical LV sustained high non-uniform stress, while the apex did not contract properly.

The signal curves for each ventricle geometry shown in figure 3 are presented in figure 4, while calculated sphericity and conicity indices are presented in table 1 .

The time-courses of haemodynamic variables for these three ventricles are shown in figure 5. In spite of the changes in geometry described above, the stroke volumes of the LV at the "normal" level of preload (end-diastolic LV pressure of $5.3 \mathrm{~mm} \mathrm{Hg}$ ) were approximately 


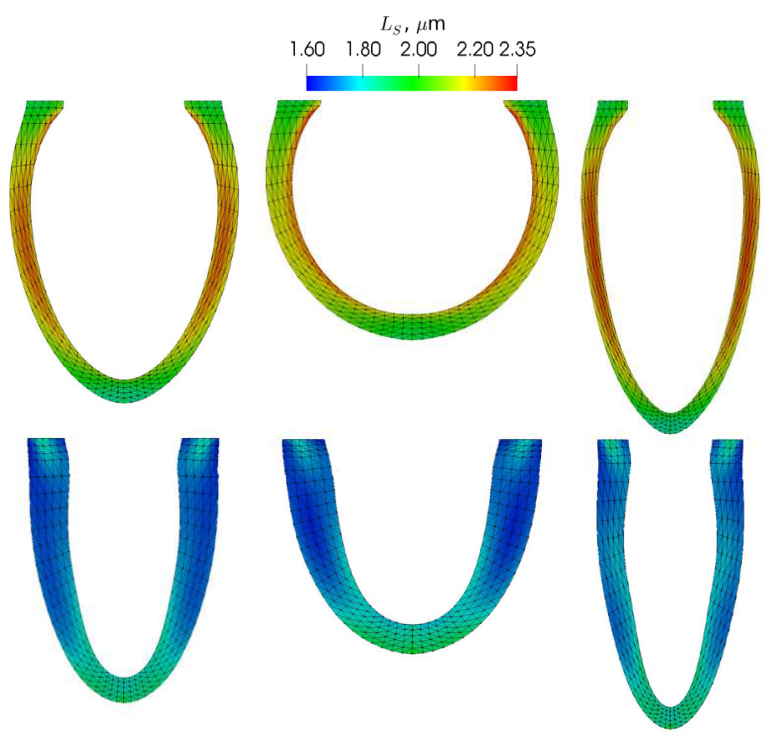

Figure 3. The end-diastolic (top) and end-systolic (bottom) geometry and sarcomere length for the "normal" (left), spherical (middle) and conical (right) LVs

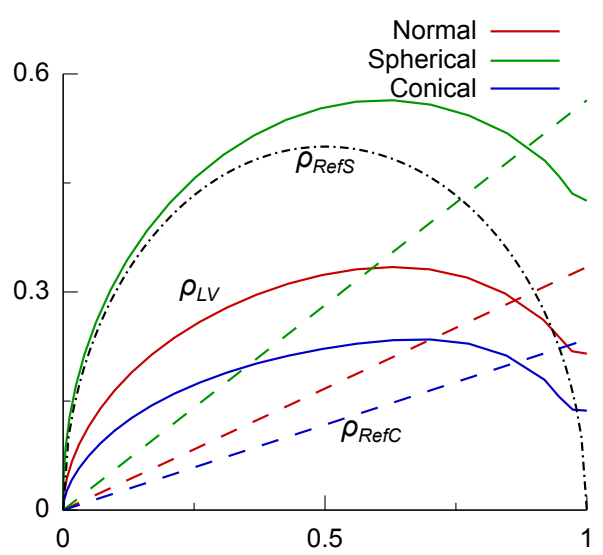

Figure 4. Shape signal curves. Solid lines are the signals for the LVs with "normal", spherical, and conical shapes. Dotted lines are the reference conical shape signals, and the dash-dotted line is the reference spherical signal

preserved (81 mL for the "normal" $\mathrm{LV}, 78 \mathrm{~mL}$ for the spherical $\mathrm{LV}$, and $85 \mathrm{~mL}$ for the conical LV), as well as ejection fractions (64\% for the "normal" LV, $60 \%$ for the spherical LV, 63\% for the conical LV). The systolic aortic pressures were slightly increased for the conical LV (126 mm Hg), and decreased for the spherical one (117 mm Hg) compared to the "normal" $\mathrm{LV}$ $(122 \mathrm{~mm} \mathrm{Hg})$. We believe that the decreases in stroke volume observed in spherical LVs after SVR reported in $[7,8]$ could be caused by the decrease in the mass of the active myocardium in ventricular wall after surgery. Also we should notice that again, as in simulations of MI, we did not consider redistribution of the orientation of muscle fibres through the ventricular 

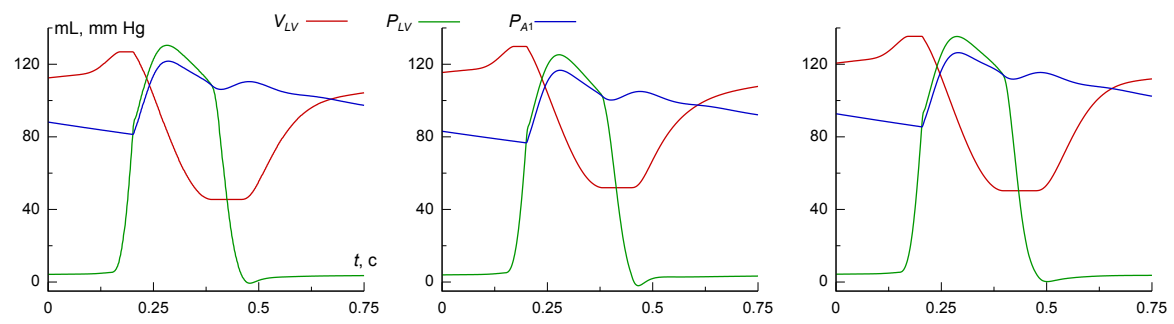

Figure 5. Simulated haemodynamics of the LV with different shape. The time-courses of the LV volume, pressure, and aortic pressure for the "normal" LV (left), spherical LV (middle), and conical LV (right) are presented

wall that was reported to have significant effects on the performance of dilated spherical LVs [6].

\section{Conclusions}

Our simulation of apical LV MI agrees with clinical data showing a reduction in the stroke volume and ejection fraction after MI involving $15 \%$ of LV surface in the apical region [14]. A substantial difference between the end-systolic and early diastolic LV shape is another feature of the simulated MI. Importantly, the simulation predicts a significant change in longitudinal strain of the LV apex and in the shape of the LV cavity during isovolumic relaxation (figure 1). Movement of the LV wall during isovolumic relaxation was suggested to be an independent predictor of heart failure [16].

The results of our simulations showed that changes in the LV shape at the constant mass of its wall did not cause any significant reduction in stroke volume and ejection fraction if the distribution of fibre orientation within the wall remained similar to that in the "healthy" LV. On the other hand, the distribution changes upon LV dilatation so that the helical angle of the subendocardial and subepicardial fibres decreases [6]. As this phenomenon was not considered in our simulations, further work is needed to simulate the effect of the distribution on the LV function.

The reported study was funded by RFBR according to the research project \# 18-31-00065.

\section{References}

[1] N. A. Trayanova, J. Constantino, V. Gurev, Am J Physiol Heart Circ Physiol 301(2), H279-86 (2011)

[2] K. Mangion, H. Gao, D. Husmeier, et al., Heart 104(7), 550-557 (2018)

[3] F. A. Syomin, M. V. Zberia, and A. K. Tsaturyan, International Journal for Numerical Methods in Biomedical Engineering $\mathbf{3 2 1 6}$ (2019)

[4] F. A. Syomin and A. K. Tsaturyan, Russian Journal of Numerical Analysis and Mathematical Modelling 32(5), 327-337 (2017)

[5] F. A. Syomin and A. K. Tsaturyan, Journal of Theoretical Biology 420, 105-116 (2017)

[6] G. Buckberg, C. Athanasuleas, J. Conte, Nat Rev Cardiol 9, 703-716 (2012)

[7] Y. Wang, C. Q. Gao, G. Wang, Y. S. Shen, Chin Med J 130, 1429-1434 (2017)

[8] L. Ch. Lee, J. F. Wenk, L. Zhong, et al., J Appl Physiol 115, 136-144 (2013)

[9] R. H. Jones, E. J. Velazquez, R. E. Michle, et al., N Engl J Med 360, 1705-1717 (2009) 
[10] F. Maffessanti, R. M. Lang, C. Corsi,x V. Mor-Avi, and E. G. Caiani, Ultrasound in Med. \& Biol. 35(12), 1953-1962 (2009)

[11] S. F. Pravdin, V. I. Berdyshev, A. V. Panfilov, et al., Biomed Eng Online 12, 54 (2013)

[12] D. Medvedofsky, K. Kebed,L. Laffin, et al, Echocardiography 34, 365-370 (2017)

[13] S. Karlsen, T. Dahlslett, B. Grenne, et al., Cardiovasc Ultrasound 17, 18 (2019)

[14] G. Joseph, T. Zaremba, M. B. Johansen, et al., Echo Res Pract 6(4), 81-89 (2019)

[15] D. Mojsejenko, J. R. McGarvey, S. M. Dorsey, et al. Biomech Model Mechanobiol 14, $633(2015)$

[16] P. Brainin, S. Haahr-Pedersen, M. Sengeløv, et al., Int J Cardiovasc Imaging 34, 751$760(2018)$ 